\title{
UNA CITA DEL ALGAZEL EN LA FILOSOFía DEL SENTIDO COMÚN DE LLORENS Y BARBA
}

\author{
Misericordia Anglés Cervelló \\ Universidad de Barcelona
}

A menudo ha sido relacionado Algazel (Abu Hamid Muhammad al-Gazali, 1058-1111) con filósofos occidentales como San Agustín, Descartes, Pascal, Hume y Kant en algunos puntos de sus respectivas filosofías.

Aquí no tratamos de establecer una relación entre el filósofo árabe y un filósofo occidental; partimos de la relación que establecen los filósofos del șentido común, el catalán Francisco J. Llorens y Barba (1820-1872) y William Hamilton (1788-1856), entre sus filosofías y la de Algazel.

Puede parecer exagerada la relación que vamos a poner de relieve entre esta filosofía del sentido común y la de Algazel, porque se trata de citas muy breves. Sin embargo, el tema en cuestión no es secundario: la noción de creencia.

Francisco J. Llorens y Barba cita la frase de Algazel «la fe es la raíz del conocimiento» después de afirmar que el conocimiento humano se apoya en actos de creencia, tesis fundamental de su filosofía del sentido común:

«De manera que unos implícita, otros explícitamente, han reconocido un lema que lo es precisamente de un filósofo arábigo, Algazel de Bagdag: radix congnitionis fides». ${ }^{1}$

La filosofía de Llorens y Barba parte claramente de los escoceses del sentido común, especialmente de William Hamilton. Este se preocupó de buscar los precedentes históricos que una filosofía del sentido común debe tener, puesto que tiene que caracterizarse por su

1 Llorens y Barba, F. J., Lecciones de Filosofía (3 vols.), Barcelona, Elzeviriana, 1920, II, p. 193. 
universalidad. En esta breve historia del sentido común encontramos la referencia a Algazel que debió ser la base de la cita de Llorens y Barba. ${ }^{2}$

Pero, aunque la cita de Hamilton sea anterior a la de Llorens y Barba, es menos explícita que la de éste. Veamos cómo Llorens explica dicha cita:

«Este filósofo famoso reconoció que, en último resultado, el conocimiento humano se apoya en una confianza innata, primitiva, ingénita, llámase como se quiera, que el hombre encuentra en sí, y de la cual el hombre no debe desposeerse, o mejor diremos, de la cual el hombre radicalmente no puede desposeerse, la cual no debe minorar si quiere juzgar rectamente». ${ }^{3}$

Algazel reconoció la importancia de la creencia dentro del conocimiento, tal y como de forma implícita o explícita debe aceptar todo filósofo. Esto es así porque existen unas creencias primitivas comunes a todo hombre por el hecho de ser hombre, de las cuales es imposible prescindir.

Aunque esta creencia no debe interpretarse como religiosa, lo cierto es que Llorens utiliza una noción muy amplia de la palabtra creencia. Así, defiende la máxima de San Anselmo «crede ut intelligas», $\mathrm{y}$, por contra, acusa de racionalista y causadora de extravío a la máxima de Abelardo «Intellige ut credas».4

No encontramos en Llorens y Barba ni en Hamilton más referencia a la creencia en Algazel. Vayamos, pues, a su misma obra y veamos qué hay en su filosofía que puediera dar pie a la filosofía del sentido común para invcarlo como un precedente.

La certeza, concebida como creencia, es realmente un tema central en la filosofía y la vida de Algazel. El mismo nos explica en su Liberación del error (al-Mungidh mina 'l-dalāl) cómo la búsqueda de la certeza fue un móvil constante en su vida. Sabemos que a los 36 años se planteó vitalmente el tema de la certeza hasta el punto de abandonar la enseñanza y hasta su familia, peregrinando durante diez años, consagrándose a la meditación sufí hata superar la crisis y retornar a la enseñanza.

La búsqueda de la certeza no puede separarse del contexto que Algazel presupone, el de la fe musulmana. No se puede considerar la cuestión de la certeza como un problema criteriológico de teoría del conocimiento sin más.

Así, el problema del que parte es el de la multiplicidad de grupos religiosos, convencidos todos ellos de estar en la verdad. Observa que los niños creen en la religión en la que han sido educados, y al mismo tiempo recuerda la frase del Profeta: «Todo hombre nace de naturaleza

2 Hamilton, W., «On the philosophy of common sense: or, our primary beliefs considered as the ultimate criterion of truth» en: Reid, T. Philosophical Works. With notes and supplementary dissertation by Sir W. Hamilton, Edimburgh, Longmans, 1895 (reimpr., Georg Olms, Hildesheim, 1983), p. 776b: «Algazel of Bagdag, "the Imaum of the World" somewhere (in his destruction of the Philosophers, if I recollect aright) says, as the Latin version gives it "radix cognitionis fides"».

3 Ibid., pp. 193-194.

4 Ibid., p. 195. 
sana, son sus progenitores quienes hacen de él un judío, un cristiano o un mazdeísta». Por eso busca Algazel la certeza originada en la misma naturaleza, porque aquella concidirá con la fe musulmana:

«Una fuerza interior me empujaba a investigar la autenticidad de la naturaleza original y la de las creencias salidas del conformismo de los progenitores y de los maestros». ${ }^{5}$

Empieza antonces un examen de sus conocimientos y no encuentra como ciertos más que las evidencias de los sentidos y de la razón. En un segundo momento llega a dudar de los datos sensibles, ya que en ciertas situaciones la razón nos hace ver que nos inducen a error. El proceso de duda sigue avanzando cuando se plantea la posibilidad de que la razón también se equivoque incluso en juicios como «diez es mayor que tres» 0 «nada de lo de aquí abajo puede ser a la vez creado y eterno, existente y no existente».

Por eso se pregunta Algazel: «¿No habrá tal vez, más allá de la razón, otro juicio cuya aparición mostrará que hay también error en la razón misma, tal como ella lo hiciera con respecto a los sentidos? Que esa inteligencia no se manifieste, no prueba que sea imposible...». ${ }^{6}$

Algazel tiene la sospecha de que el estado de vigilia tal vez sea una cierta forma de sueño, que toda la vida sea un sueño del que se despierta con la muerte. Algo parecido al estado místico de los sufís.

Este proceso, que nos hace pensar en la duda cartesiana, no se supera con ninguna argumentación racional:

«El mal empeoró y se prolongó durante dos meses, durante los cuales fui presa del 'sofisma' (safsata). Ese era mi estado de alma real, aunque nada se transparentara en mis palabras. Finalmente, Dios me curó y recuperé la salud y el equilibrio mental. Los datos racionales necesarios volvieron a ser aceptables; tuve confianza en ellos; volví a hallarme seguro y en la certeza. No llegué allí mediante razonamientos bien ordenados no discursos metódicamente construídos, sino por medio de una luz que Dios proyectó en mi pecho». ${ }^{?}$

Algazel se refiere a menudo al «corazón» como una facultad de conocimiento, o también como un conocimiento superior. La plenitud de la certeza, que es la buscada en este texto, se da más en el corazón que en la razón. La certeza no tiene su origen en la argumentación metódica. La certeza debe entenderse como la verdad dentro del Islam.

5 Algazel, Liberation del error (al-Munguidh mina 'l-daläl), Rosario, E. Peregrino, 1983, p. 29.

6 Ibid., p. 34.

7 Ibid., p. 35 . 
Lo importante no es el medio por el que se adquiere la certeza, sino el resultado obtenido.

«En el momento que la imagen de lo verdadero se graba en el corazón no importa qué causa ha contribuído a grabarla. Esta puede ser un argumento verdadero, o recibido comúnmente, o por simple conveniencia, puede ser la aceptación basada en la creencia en el que enseña o bien la aceptación por simple submisión a una enseñanza transmitida». 8

Algazel distingue y admite dos significacados distintos de la palabra certeza (yaqyuin). La primera se refiere al saber especulativo y se define simplemente como la ausencia de duda. En relación a ella Algazel distingue cuatro grados. En primer lugar está la duda, es decir, cuando hay igualdad de fuerza para creer como para no creer. Después se da el estado en que la fuerza está en una de las dos soluciones, pero con la conciencia de la posibilidad de lo contrario. El tercer grado consiste en una creencia, porque al faltar la verificación deja lugar para la duda.

Hay un segundo sentido de la certeza, aquélla que poseen muchos sabios y sufís. Es un convencimiento radicado en la razón y en el corazón, y que influye en la conducta de la persona. Todos los hombres poseen el primer tipo de certeza acerca de que hay que morir, pues es difícil dudar de ello. Sin embargo, muchas personas carecen del segundo tipo de certeza, puesto que no son conscientes de su inevitable mortalidad, y viven como si lo ignorasen. Esta certeza conlleva una mayor perfección.

Dentro de esta certeza también esisten grados que surgen según la intensidad de tal certeza. ${ }^{9}$

Después de haber superado el estado de duda, Algazel hace referencia a las primeras nociones. Estas pueden ser proposiciones muy distinas entre sí, desde el conocimiento de la propia existencia, hasta la proposición «dos es más que uno», o también «una misma cosa no puede ser a la vez antigua y estar empezando a existir». Tienen en común el darse en la razón por su propia naturaleza, existiendo en ella desde siempre y necesariamente. Son primeras porque se dan de forma inmediata. No se pueden considerar primeros principios porque se trata más bien de conocimientos concretos e individuales. Tampoco se caracterizan por su universalidad sino por su firme certeza inmutable. Se constatan como un hecho, independientemente de que se pueda explicar o no su procedencia.

Es más, no hay que buscar su origen, puesto que su forma de ser es la presencia en la razón:

«No hay que buscar las nociones primeras, puesto que éstas están presentes en el espíritu. Lo que está presente desaparece cuando se lo busca. No podrá acusarse de negligente a aquél que se pone a buscar lo que no le es preciso buscar».. ${ }^{10}$

8 Iljam al-'awamm an ilm al-kalam, 56-59, vid. Annexe II en Jabre, Farid, La notion de certitude selon Ghazali, Paris, Vrin, 1958.

9 Algazel distingue estos dos tipos de certeza en Ihya 'Ulum ad-din. Los textos referidos a ello se pueden encontrar en el Annexe IV, p. 436 en Jabre, Farid, La notion de certitude selon Ghazali, Vrin, Paris, 1958.

10 al-Munguidh mina ' $l$-dalal, p. 36. 
Las características de estas nociones primeras coinciden con las que definen las primeras creencias tanto en Hamilton como en Llorens y Barba.

Algazel clasifica con el mismo tipo de certeza de estas verdades el conocimiento de Dios y el de la veracidad del Profeta. Todo ello se impone con la misma fuerza al sujeto cognoscente, aunque las verdades necesarias tienen una función propedéutica respecto de las otras. Son las primeras en darse y nos preparan al concimiento de Dios.

Después de esta visión sintética de la certeza en Algazel, ¿podemos concluir afirmando que es correcto considerar al filósofo árabe como un precedente de la filosofía del sentido común?

Sería ciertamente exagerado denominar a Algazel filósofo del sentido común. Su preocupación central fue la defensa de la fe, y por ello llegó a cuestionar críticamente la filosofía. No hay que desvincular sus textos del contexto islámico histórico en el que vivió ${ }^{11}$ adaptándolo forzadamente a los problemas de la filosofía occidental posterior.

Sin embargo, es cierto que su noción de certeza como creencia coincide con el planteamiento de las creencias primitivas de Llorens y Barba. También hay coincidencia en la valoración de un elemento no obtenido por especulación, pero esencial al conocimiento.

En este sentido parcial, se puede hablar de una relación entre Algazel y la filosofía del sentido común.

11 Jabre, F., La notion de certitude selon Ghazali dans ses origines phychologiques et historiques, Vrin, Paris, 1958. 